\title{
Analysis of $\mathrm{SO}_{2}$ Physisorption by Edge-Functionalized Nanoporous Carbons Using Grand Canonical Monte Carlo Methods and Density Functional Theory: Implications for $\mathrm{SO}_{2}$ Removal.
}

\author{
Ruyi Zhao ${ }^{1}$, Guodong Liu ${ }^{1 *}$, Guohua Wei ${ }^{1,2,3}$, Jihui Gao ${ }^{1}$, Huilin $\mathrm{Lu}^{1}$
}

1. School of Energy Science and Engineering, Harbin Institute of Technology, Harbin, 150001, China

2. State Key Laboratory of Efficient and Clean Coal-fired Utility Boiler (Harbin Boiler Company Limited), Harbin 150046,

\section{China}

3. Harbin Boiler Company Limited, Harbin 150046, China

* Corresponding author: Guodong Liu, gdliu@hit.edu.cn 


\section{Figures}

(a)

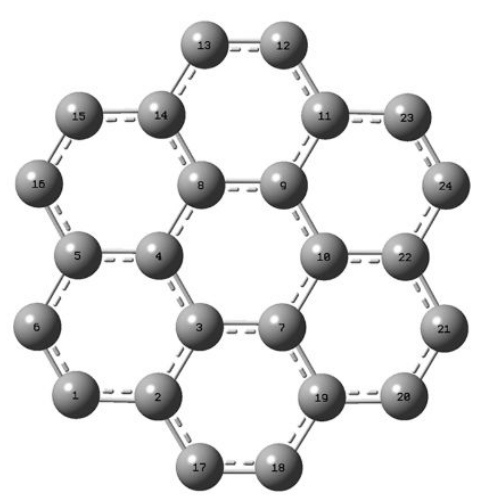

(c)

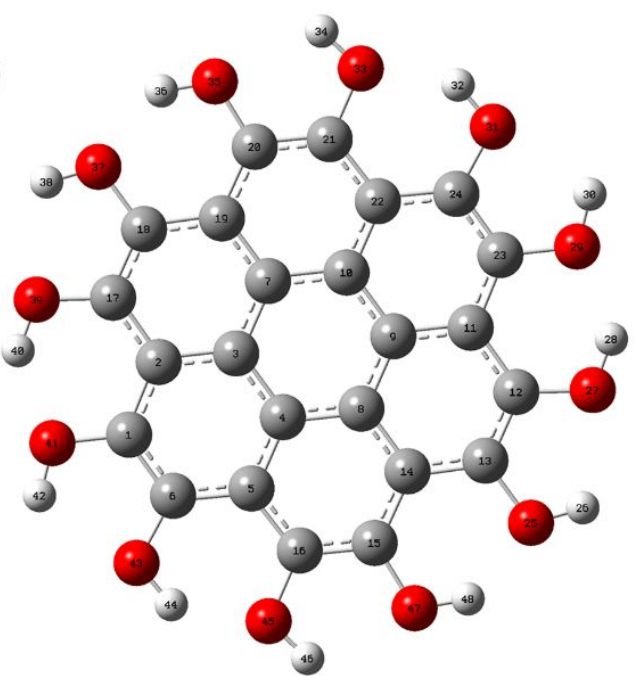

(e)

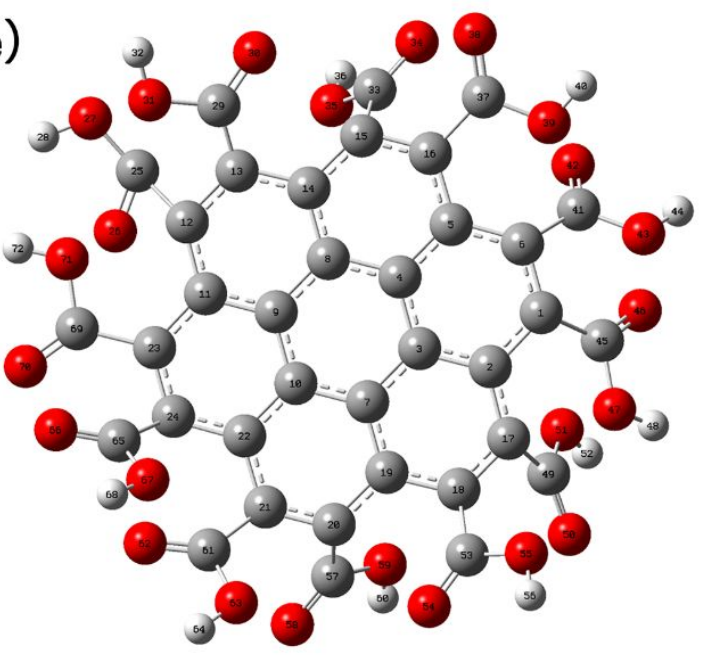

(b)
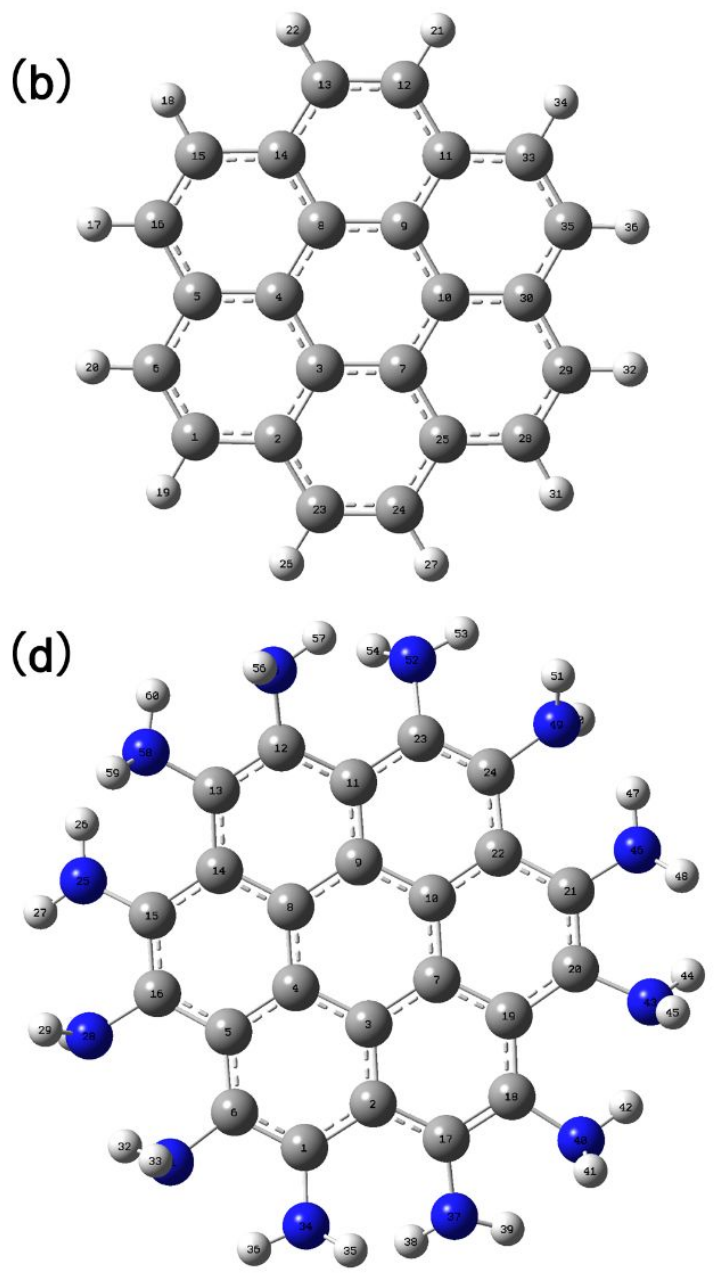

Figure S1.The marked BSUs. (a)BSU; (b)BSU-H; (c)BSU-OH; (d)BSU-NH ${ }_{2}$; (e)BSU-COOH 

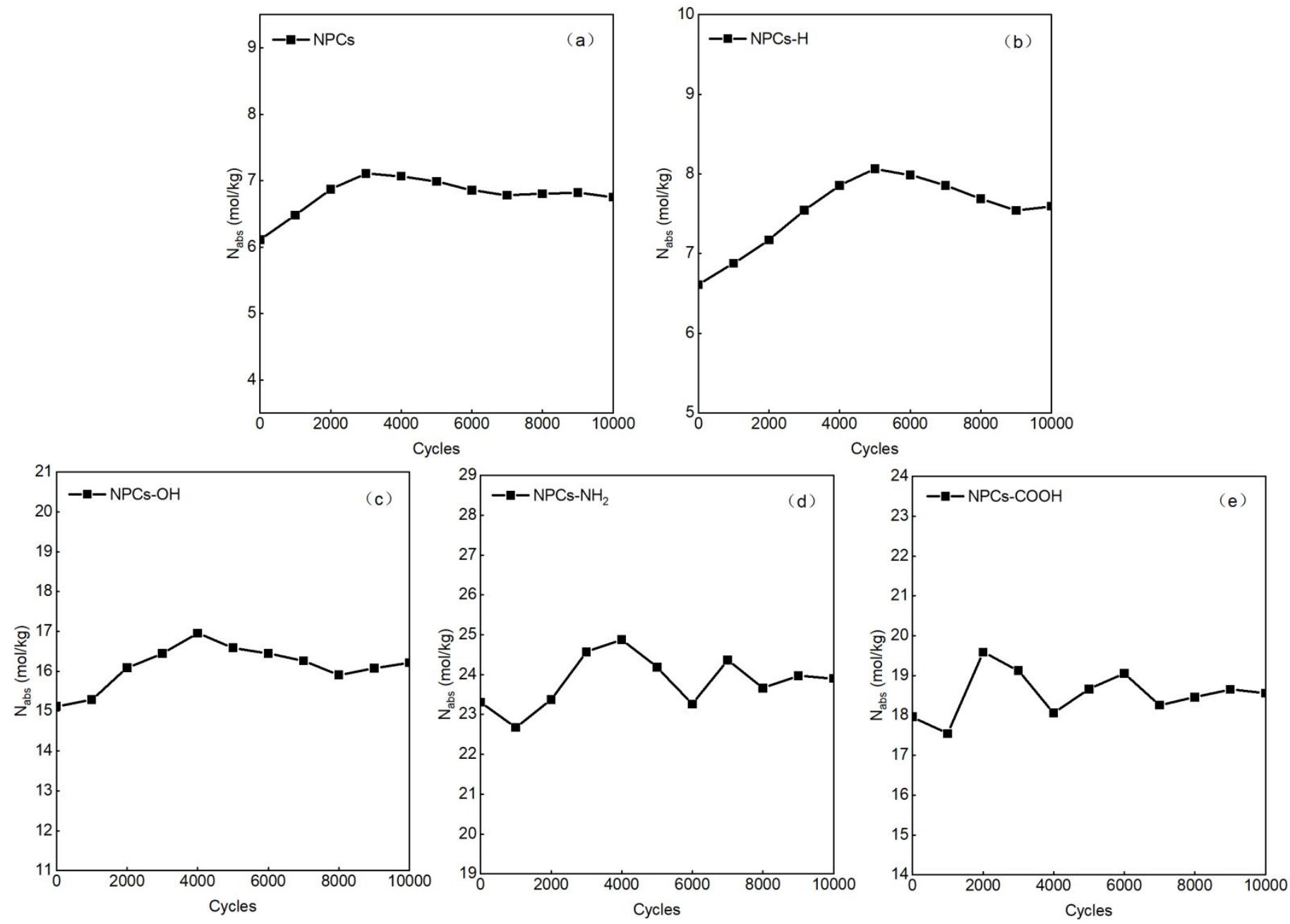

Figure S2. The number of adsorbed molecules versus cycles at $\mathrm{T}=298 \mathrm{~K}$ and $\mathrm{P}=220 \mathrm{kPa}$. (a)NPCs; (b)NPCs$\mathrm{H}$; (c)NPCs-OH; (d)NPCs- $\mathrm{NH}_{2}$; (e)NPCs-COOH 


\section{Table}

Table S1.Atomic partial charges by RESP charges analysis

\begin{tabular}{|c|c|c|c|c|c|c|c|c|c|c|c|c|c|c|}
\hline \multicolumn{3}{|c|}{ BSU-None } & \multicolumn{3}{|c|}{ BSU-H } & \multicolumn{3}{|c|}{ BSU-OH } & \multicolumn{3}{|c|}{ BSU-NH ${ }_{2}$} & \multicolumn{3}{|c|}{ BSU-COOH } \\
\hline 1 & $\mathrm{C}$ & -0.024742 & 1 & $\mathrm{C}$ & -0.217963 & 1 & $\mathrm{C}$ & 0.150591 & 1 & $\mathrm{C}$ & 0.334077 & 1 & $\mathrm{C}$ & -0.365469 \\
\hline 2 & $\mathrm{C}$ & 0.075417 & 2 & $\mathrm{C}$ & 0.119160 & 2 & $\mathrm{C}$ & -0.251932 & 2 & $\mathrm{C}$ & -0.283350 & 2 & $\mathrm{C}$ & 0.273789 \\
\hline 3 & $\mathrm{C}$ & -0.025682 & 3 & $\mathrm{C}$ & -0.006208 & 3 & $\mathrm{C}$ & 0.017955 & 3 & $\mathrm{C}$ & -0.080937 & 3 & $\mathrm{C}$ & -0.036223 \\
\hline 4 & $\mathrm{C}$ & -0.024954 & 4 & $\mathrm{C}$ & -0.006711 & 4 & $\mathrm{C}$ & 0.019396 & 4 & $\mathrm{C}$ & 0.133292 & 4 & $\mathrm{C}$ & 0.055140 \\
\hline 5 & $\mathrm{C}$ & 0.074636 & 5 & $\mathrm{C}$ & 0.123649 & 5 & $\mathrm{C}$ & -0.259697 & 5 & $\mathrm{C}$ & -0.431012 & 5 & $\mathrm{C}$ & 0.167446 \\
\hline 6 & $\mathrm{C}$ & -0.026272 & 6 & $\mathrm{C}$ & -0.224978 & 6 & $\mathrm{C}$ & 0.342552 & 6 & $\mathrm{C}$ & 0.303354 & 6 & $\mathrm{C}$ & -0.193865 \\
\hline 7 & $\mathrm{C}$ & -0.017432 & 7 & $\mathrm{C}$ & -0.008638 & 7 & $\mathrm{C}$ & 0.013632 & 7 & $\mathrm{C}$ & 0.066726 & 7 & $\mathrm{C}$ & 0.030048 \\
\hline 8 & $\mathrm{C}$ & -0.017575 & 8 & $\mathrm{C}$ & -0.008327 & 8 & $\mathrm{C}$ & 0.021274 & 8 & $\mathrm{C}$ & -0.044837 & 8 & $\mathrm{C}$ & -0.000714 \\
\hline 9 & $\mathrm{C}$ & -0.026212 & 9 & $\mathrm{C}$ & -0.004071 & 9 & $\mathrm{C}$ & 0.013629 & 9 & $\mathrm{C}$ & 0.040913 & 9 & $\mathrm{C}$ & -0.070911 \\
\hline 10 & $\mathrm{C}$ & -0.028935 & 10 & $\mathrm{C}$ & -0.005476 & 10 & $\mathrm{C}$ & 0.020542 & 10 & $\mathrm{C}$ & 0.000066 & 10 & $\mathrm{C}$ & -0.002014 \\
\hline 11 & $\mathrm{C}$ & 0.077460 & 11 & $\mathrm{C}$ & 0.118978 & 11 & $\mathrm{C}$ & -0.253312 & 11 & $\mathrm{C}$ & -0.300041 & 11 & $\mathrm{C}$ & 0.330593 \\
\hline 12 & $\mathrm{C}$ & -0.025535 & 12 & $\mathrm{C}$ & -0.219398 & 12 & $\mathrm{C}$ & 0.341139 & 12 & $\mathrm{C}$ & 0.293823 & 12 & $\mathrm{C}$ & -0.331659 \\
\hline 13 & $\mathrm{C}$ & -0.024405 & 13 & $\mathrm{C}$ & -0.222193 & 13 & $\mathrm{C}$ & 0.156550 & 13 & $\mathrm{C}$ & 0.233918 & 13 & $\mathrm{C}$ & -0.296369 \\
\hline 14 & $\mathrm{C}$ & 0.068925 & 14 & $\mathrm{C}$ & 0.122727 & 14 & $\mathrm{C}$ & -0.257867 & 14 & $\mathrm{C}$ & -0.215356 & 14 & $\mathrm{C}$ & 0.194892 \\
\hline 15 & $\mathrm{C}$ & -0.024647 & 15 & $\mathrm{C}$ & -0.222651 & 15 & $\mathrm{C}$ & 0.337520 & 15 & $\mathrm{C}$ & 0.240324 & 15 & $\mathrm{C}$ & -0.286232 \\
\hline 16 & $\mathrm{C}$ & -0.024890 & 16 & $\mathrm{C}$ & -0.220850 & 16 & $\mathrm{C}$ & 0.160669 & 16 & $\mathrm{C}$ & 0.223902 & 16 & $\mathrm{C}$ & -0.460769 \\
\hline 17 & $\mathrm{C}$ & -0.026409 & 17 & $\mathrm{H}$ & 0.163574 & 17 & $\mathrm{C}$ & 0.335478 & 17 & $\mathrm{C}$ & 0.323818 & 17 & $\mathrm{C}$ & -0.392046 \\
\hline 18 & $\mathrm{C}$ & -0.023741 & 18 & $\mathrm{H}$ & 0.164138 & 18 & $\mathrm{C}$ & 0.158954 & 18 & $\mathrm{C}$ & 0.332091 & 18 & $\mathrm{C}$ & -0.036498 \\
\hline 19 & $\mathrm{C}$ & 0.069981 & 19 & $\mathrm{H}$ & 0.162831 & 19 & $\mathrm{C}$ & -0.253350 & 19 & $\mathrm{C}$ & -0.376478 & 19 & $\mathrm{C}$ & -0.053716 \\
\hline 20 & $\mathrm{C}$ & -0.026198 & 20 & $\mathrm{H}$ & 0.164873 & 20 & $\mathrm{C}$ & 0.339688 & 20 & $\mathrm{C}$ & 0.214110 & 20 & $\mathrm{C}$ & -0.217419 \\
\hline 21 & $\mathrm{C}$ & -0.024120 & 21 & $\mathrm{H}$ & 0.163527 & 21 & $\mathrm{C}$ & 0.152384 & 21 & $\mathrm{C}$ & 0.221213 & 21 & $\mathrm{C}$ & -0.409185 \\
\hline 22 & $\mathrm{C}$ & 0.079930 & 22 & $\mathrm{H}$ & 0.164004 & 22 & $\mathrm{C}$ & -0.252769 & 22 & $\mathrm{C}$ & -0.258912 & 22 & $\mathrm{C}$ & 0.199018 \\
\hline 23 & $\mathrm{C}$ & -0.024957 & 23 & $\mathrm{C}$ & -0.218840 & 23 & $\mathrm{C}$ & 0.151937 & 23 & $\mathrm{C}$ & 0.247162 & 23 & $\mathrm{C}$ & -0.531210 \\
\hline \multirow[t]{17}{*}{24} & $\mathrm{C}$ & -0.029638 & 24 & $\mathrm{C}$ & -0.223059 & 24 & $\mathrm{C}$ & 0.341322 & 24 & $\mathrm{C}$ & 0.226986 & 24 & $\mathrm{C}$ & -0.291002 \\
\hline & & & 25 & $\mathrm{C}$ & 0.124570 & 25 & $\mathrm{O}$ & -0.464685 & 25 & $\mathrm{~N}$ & -0.799419 & 25 & $\mathrm{C}$ & 0.790403 \\
\hline & & & 26 & $\mathrm{H}$ & 0.163317 & 26 & $\mathrm{H}$ & 0.383547 & 26 & $\mathrm{H}$ & 0.335931 & 26 & $\mathrm{O}$ & -0.243152 \\
\hline & & & 27 & $\mathrm{H}$ & 0.164099 & 27 & $\mathrm{O}$ & -0.576572 & 27 & $\mathrm{H}$ & 0.350770 & 27 & $\mathrm{O}$ & -0.732797 \\
\hline & & & 28 & $\mathrm{C}$ & -0.223764 & 28 & $\mathrm{H}$ & 0.398739 & 28 & $\mathrm{~N}$ & -0.502879 & 28 & $\mathrm{H}$ & 0.529533 \\
\hline & & & 29 & $\mathrm{C}$ & -0.218038 & 29 & $\mathrm{O}$ & -0.463166 & 29 & $\mathrm{H}$ & 0.223712 & 29 & $\mathrm{C}$ & 0.767816 \\
\hline & & & 30 & $\mathrm{C}$ & 0.118076 & 30 & $\mathrm{H}$ & 0.383299 & 30 & $\mathrm{H}$ & 0.258081 & 30 & $\mathrm{O}$ & -0.315156 \\
\hline & & & 31 & $\mathrm{H}$ & 0.164097 & 31 & $\mathrm{O}$ & -0.576318 & 31 & $\mathrm{~N}$ & -0.848779 & 31 & $\mathrm{O}$ & -0.715695 \\
\hline & & & 32 & $\mathrm{H}$ & 0.162972 & 32 & $\mathrm{H}$ & 0.398662 & 32 & $\mathrm{H}$ & 0.270027 & 32 & $\mathrm{H}$ & 0.521529 \\
\hline & & & 33 & $\mathrm{C}$ & -0.221978 & 33 & $\mathrm{O}$ & -0.463465 & 33 & $\mathrm{H}$ & 0.346184 & 33 & $\mathrm{C}$ & 1.059264 \\
\hline & & & 34 & $\mathrm{H}$ & 0.164150 & 34 & $\mathrm{H}$ & 0.383395 & 34 & $\mathrm{~N}$ & -0.853178 & 34 & $\mathrm{O}$ & -0.661184 \\
\hline & & & 35 & $\mathrm{C}$ & -0.219106 & 35 & $\mathrm{O}$ & -0.575412 & 35 & $\mathrm{H}$ & 0.391763 & 35 & $\mathrm{O}$ & -0.764402 \\
\hline & & & 36 & $\mathrm{H}$ & 0.163511 & 36 & $\mathrm{H}$ & 0.398323 & 36 & $\mathrm{H}$ & 0.334587 & 36 & $\mathrm{H}$ & 0.490046 \\
\hline & & & & & & 37 & $\mathrm{O}$ & -0.465164 & 37 & $\mathrm{~N}$ & -0.915535 & 37 & $\mathrm{C}$ & 1.031736 \\
\hline & & & & & & 38 & $\mathrm{H}$ & 0.383750 & 38 & $\mathrm{H}$ & 0.400931 & 38 & $\mathrm{O}$ & -0.668656 \\
\hline & & & & & & 39 & $\mathrm{O}$ & -0.574567 & 39 & $\mathrm{H}$ & 0.362870 & 39 & $\mathrm{O}$ & -0.717426 \\
\hline & & & & & & 40 & $\mathrm{H}$ & 0.396676 & 40 & $\mathrm{~N}$ & -0.886390 & 40 & $\mathrm{H}$ & 0.496008 \\
\hline
\end{tabular}




\begin{tabular}{|c|c|c|c|c|c|c|c|c|}
\hline 41 & $\mathrm{O}$ & -0.458544 & 41 & $\mathrm{H}$ & 0.345261 & 41 & $\mathrm{C}$ & 1.019064 \\
\hline 42 & $\mathrm{H}$ & 0.381059 & 42 & $\mathrm{H}$ & 0.297954 & 42 & $\mathrm{O}$ & -0.617809 \\
\hline 43 & $\mathrm{O}$ & -0.575909 & 43 & $\mathrm{~N}$ & -0.507679 & 43 & $\mathrm{O}$ & -0.744232 \\
\hline 44 & $\mathrm{H}$ & 0.399358 & 44 & $\mathrm{H}$ & 0.217511 & 44 & $\mathrm{H}$ & 0.490022 \\
\hline 45 & $\mathrm{O}$ & -0.467041 & 45 & $\mathrm{H}$ & 0.257800 & 45 & $\mathrm{C}$ & 0.931948 \\
\hline 46 & $\mathrm{H}$ & 0.384452 & 46 & $\mathrm{~N}$ & -0.724982 & 46 & $\mathrm{O}$ & -0.570171 \\
\hline 47 & $\mathrm{O}$ & -0.576332 & 47 & $\mathrm{H}$ & 0.267948 & 47 & $\mathrm{O}$ & -0.828341 \\
\hline \multirow[t]{25}{*}{48} & $\mathrm{H}$ & 0.399624 & 48 & $\mathrm{H}$ & 0.332748 & 48 & $\mathrm{H}$ & 0.532676 \\
\hline & & & 49 & $\mathrm{~N}$ & -0.624778 & 49 & $\mathrm{C}$ & 0.728429 \\
\hline & & & 50 & $\mathrm{H}$ & 0.303378 & 50 & $\mathrm{O}$ & -0.307306 \\
\hline & & & 51 & $\mathrm{H}$ & 0.306873 & 51 & $\mathrm{O}$ & -0.783529 \\
\hline & & & 52 & $\mathrm{~N}$ & -0.800645 & 52 & $\mathrm{H}$ & 0.556137 \\
\hline & & & 53 & $\mathrm{H}$ & 0.310915 & 53 & $\mathrm{C}$ & 0.750783 \\
\hline & & & 54 & $\mathrm{H}$ & 0.384827 & 54 & $\mathrm{O}$ & -0.390906 \\
\hline & & & 55 & $\mathrm{~N}$ & -0.903398 & 55 & $\mathrm{O}$ & -0.407315 \\
\hline & & & 56 & $\mathrm{H}$ & 0.383115 & 56 & $\mathrm{H}$ & 0.480546 \\
\hline & & & 57 & $\mathrm{H}$ & 0.330211 & 57 & $\mathrm{C}$ & 0.798373 \\
\hline & & & 58 & $\mathrm{~N}$ & -0.743811 & 58 & $\mathrm{O}$ & -0.330879 \\
\hline & & & 59 & $\mathrm{H}$ & 0.354536 & 59 & $\mathrm{O}$ & -0.687232 \\
\hline & & & 60 & $\mathrm{H}$ & 0.298677 & 60 & $\mathrm{H}$ & 0.524290 \\
\hline & & & & & & 61 & $\mathrm{C}$ & 0.834056 \\
\hline & & & & & & 62 & $\mathrm{O}$ & -0.241905 \\
\hline & & & & & & 63 & $\mathrm{O}$ & -0.750738 \\
\hline & & & & & & 64 & $\mathrm{H}$ & 0.550099 \\
\hline & & & & & & 65 & $\mathrm{C}$ & 1.102915 \\
\hline & & & & & & 66 & $\mathrm{O}$ & -0.702944 \\
\hline & & & & & & 67 & $\mathrm{O}$ & -0.743323 \\
\hline & & & & & & 68 & $\mathrm{H}$ & 0.499748 \\
\hline & & & & & & 69 & $\mathrm{C}$ & 1.053862 \\
\hline & & & & & & 70 & $\mathrm{O}$ & -0.630247 \\
\hline & & & & & & 71 & $\mathrm{O}$ & -0.750770 \\
\hline & & & & & & 72 & $\mathrm{H}$ & 0.491211 \\
\hline
\end{tabular}

